\title{
Defect indices of singular symmetric linear difference equations with complex coefficients
}

\author{
Guojing Ren
}

Correspondence: rgjmaths@gmail. com

School of Mathematics and Quantitative Economics, Shandong Provincial Key Laboratory of Digital Media Technology, Shandong University of Finance and Economics, Jinan, Shandong 250014, P. R. China

\section{Abstract}

This article is concerned with the defect indices of singular symmetric linear difference equations of order $2 n$ with complex coefficients and one singular endpoint. We first show that the positive and negative defect indices $d_{+}$and $d_{-}$of a class of singular symmetric linear difference equations of order $2 n$ with complex coefficients satisfy the inequalities $n \leq d_{+}=d_{-} \leq 2 n$ and all values of this range are realized. This extends the result for difference equations with real coefficients. In addition, some sufficient conditions for the limit point and the strong limit point cases are given.

AMS Classification: 39A70; 34B20.

Keywords: singular symmetric linear difference equation, square summable solution, defect index, limit point case, strong limit point case

\section{Introduction}

In this article, we are interested in the positive and negative defect indices of the following singular symmetric linear difference equation with complex coefficients:

$$
\begin{aligned}
& \sum_{j=0}^{n}(-1)^{j} \Delta^{j}\left(p_{j}(t) \nabla^{j} y(t)\right)+i \sum_{k=1}^{n}\left[(-1)^{k+1} \Delta^{k}\left(q_{k}(t) y(t)\right)+q_{k}(t) \nabla^{k} y(t)\right] \\
& \quad=\lambda w(t) y(t), \quad t \in \mathcal{I},
\end{aligned}
$$

where $\Delta$ and $\nabla$ are forward and backward difference operators, respectively, i.e., $\Delta y(t)$ $=y(t+1)-y(t)$ and $\nabla y(t)=y(t)-y(t-1) ; \mathcal{I}:=[0,+\infty)=\{t\}_{t=0}^{+\infty} ; w(t)>0, p_{j}(t)$, and $q_{k}(t)$ are real-valued for $0 \leq j \leq n, 1 \leq k \leq n$; $p_{n}^{2}(t)+q_{n}^{2}(t) \neq 0$ for $t \in \mathcal{I}$; and $\lambda$ is a complex spectral parameter.

By letting $u(t)=\left(u_{1}(t), u_{2}(t), \ldots, u_{2 n}(t)\right)^{T}$ with

$$
\begin{aligned}
u_{j}(t) & =\Delta^{j-1} \gamma(t-j), \\
u_{n+j}(t) & =\sum_{k=j}^{n}(-1)^{k-j}\left[\Delta^{k-j}\left(p_{k}(t) \nabla^{k} y(t)-i q_{k}(t) y(t)\right)\right],
\end{aligned}
$$

for $1 \leq j \leq n$, Equation (1.1) can be converted into the following singular linear discrete Hamiltonian system

$$
J \Delta u(t)=(\lambda W(t)+P(t)) R(u)(t), \quad t \in \mathcal{I},
$$

\section{Springer}

(c) 2012 Ren; licensee Springer. This is an Open Access article distributed under the terms of the Creative Commons Attribution License (http://creativecommons.org/licenses/by/2.0), which permits unrestricted use, distribution, and reproduction in any medium, provided the original work is properly cited. 
where

$$
W(t)=\operatorname{diag}\{w(t), 0, \ldots, 0\}, \quad P(t)=\left(\begin{array}{cc}
-C(t) & A^{*}(t) \\
A(t) & B(t)
\end{array}\right)
$$

and

$$
\begin{aligned}
& A(t)=\left(\begin{array}{cc}
0 & I_{n-1} \\
i \frac{q_{n}(t)}{p_{n}(t)} & 0
\end{array}\right), \quad B(t)=\operatorname{diag}\left\{0, \ldots, 0, p_{n}^{-1}(t)\right\} \\
& C(t)=\left(\begin{array}{cc}
p_{0}(t)-\frac{q_{n}(t)}{p_{n}(t)} & \alpha(t) \\
\alpha^{*}(t) & D(t)
\end{array}\right), \\
& \alpha(t)=\left(i q_{n-1}(t), i q_{n-2}(t), \ldots, i q_{1}(t)\right), \\
& D(t)=\operatorname{diag}\left\{p_{1}(t), p_{2}(t), \ldots, p_{n-1}(t)\right\},
\end{aligned}
$$

$I_{n-1}$ is the $(n-1) \times(n-1)$ unit matrix, and the right partial shift operator

$$
R(u)(t)=\left(u_{1}(t+1), \ldots, u_{n}(t+1), u_{n+1}(t), \ldots, u_{2 n}(t)\right)^{T} .
$$

According to the classical von Neumann theory (cf. [1,2]) and its generalization [3], a symmetric operator or a Hermitian subspace has a self-adjoint extension if and only if its positive and negative defect indices are equal and its self-adjoint extension domains have a close relationship with its defect indices. So it is very important to determine the defect indices of both differential equations and difference equations in the study of self-adjoint extensions.

Consider singular symmetric linear differential equation with complex coefficients:

$$
\begin{aligned}
& \sum_{j=0}^{n}(-1)^{j}\left[p_{j}(x) y^{(j)}(x)\right]^{(j)}+i \sum_{k=0}^{n-1}\left[\left(q_{k}(t) x^{(k+1)}(t)\right)^{(k)}+\left(q_{k}(t) x^{k}(t)\right)^{(k+1)}\right] \\
& \quad=\lambda w(x) y(x), \quad x \in(a, b),
\end{aligned}
$$

where $p_{j}$ and $q_{k}$ are all real functions, and $w(t)>0$ defined on $(a, b)$. The defect indices of Equation (1.6) has been studied for a long time. It is well known that the positive and negative defect indices $d_{ \pm}$of (1.6) are equal to the number of linearly independent square integrable solutions of (1.6) with $\operatorname{Im} \lambda>0$ and $\operatorname{Im} \lambda<0$, respectively. In the special case that the coefficients of (1.6) is real; that is all $q_{k}(t) \equiv 0$, it is evident that $d_{+}=d_{-}:=d$. Glazman [4] showed that the defect index $d$ of Equation (1.6) with real coefficients defined on $(0,+\infty)$, where $x=0$ is a regular endpoint, satisfies the inequalities $n \leq d \leq 2 n$ and all values of $d$ in this range are realized. Many other results on the defect index $d$ of Equation (1.6) with real coefficients were summarized in [5]. Mcleod gave an example of a fourth-order symmetric ordinary differential equation, whose positive and negative defect indices are different [6]. Kogan and Rofe-Beketov discussed the positive and negative defect indices of Equation (1.6), and showed that the positive and negative defect indices may differ an integer $[7,8]$.

For the discrete case, Atkinson first studied the number of linearly independent square summable solutions of second-order symmetric linear difference equations with real coefficients [9]. Subsequently, his study was further developed (cf. [10-13]). Sun studied the number of linearly independent square summable solutions of secondorder symmetric difference equations with complex coefficients [14]. It has been 
shown that the positive and negative defect indices of second-order symmetric difference equations with complex coefficients are still equal; that is $\left(d_{+}, d_{-}\right)=(1,1)$ or $(2,2)$. The positive and negative defect indices of singular symmetric linear difference equation with real coefficients:

$$
\sum_{j=0}^{n}(-1)^{j} \Delta^{j}\left(p_{j}(t) \nabla^{j} \gamma(t)\right)=\lambda w(t) y(t), \quad t \in \mathcal{I},
$$

has been discussed in [15]. Since the coefficients of (1.7) are all real, it can be easily verified that $d_{+}=d_{-}:=d$. It has been shown in [15] that $n \leq d \leq 2 n$, and all values in this range can be realized. There are seldom results on the positive and negative defect indices of symmetric difference equation with complex coefficients.

In the present article, we study the positive and negative defect indices of Equation (1.1). The rest of the article is organized as follows. In Section 2, two equivalent forms of Equation (1.1) are formulated, and some useful lemmas are stated. In Section 3, we pay attention to the defect indices of Equation (1.1) and its equivalent forms. Theorem 3.1 and Example 3.1 show that the positive and negative defect indices of a class of symmetric difference equations of order $2 n$ with complex coefficients satisfies the inequalities $n \leq d_{+}=d_{-} \leq 2 n$ and all values in this range are realized. This extends the corresponding result for symmetric difference equations with real coefficients in [15]. We point out the method used here is different from that in [15]. In addition, several criteria of the limit point and strong limit point cases are established. Most of the results in the present article extend the corresponding results for real coefficient equations in [15].

\section{Preliminaries}

This section is divided into three sections. In Section 2.1, a classification of limit cases of Equation (1.1) is introduced. In Section 2.2, two equivalent forms of Equation (1.1) are introduced. In Section 2.3, some sufficient and necessary conditions of limit point case and strong limit point case of Equation (1.1) are given. Some of the results in this section can be regarded as extensions of those in [15].

\subsection{Classification of limit cases}

By $\mathbb{C}$ denotes the set of the complex numbers, and by $\bar{z}$ and $u^{*}$ denote the conjugate of $z$ and the complex conjugate transpose of $u$, respectively.

We now introduce the following space:

$$
l_{w}^{2}(\mathcal{I}):=\left\{y=\{\gamma(t)\}_{t=-n}^{+\infty} \subset \mathbb{C}: \sum_{t \in \mathcal{I}} w(t)|y(t)|^{2}<+\infty\right\}
$$

with inner product

$$
\langle x, y\rangle_{w}:=\sum_{t \in \mathcal{I}} w(t) \bar{y}(t) x(t),
$$

where the weight function $w(t)>0$ on $\mathcal{I}$. For $x, y \in l_{w}^{2}(\mathcal{I}), x$ is said to be equal to $y$ if $\|x-y\|_{w}=0$, where $\|\cdot\|_{w}:=\left(\langle\cdot, \cdot\rangle_{w}\right)^{1 / 2}$. In this sense, $l_{w}^{2}(\mathcal{I})$ is a Hilbert space with the inner product $\langle\cdot, \cdot\rangle_{w}$. In the special case of $w(t) \equiv 1, l_{w}^{2}(\mathcal{I})$ is briefly denoted by $l^{2}(\mathcal{I})$. 
Similarly to the scalar case, denote

$$
L_{w}^{2}(\mathcal{I}):=\left\{u=\{u(t)\}_{t=0}^{+\infty} \subset \mathbb{C}^{2 n}: \sum_{t \in \mathcal{I}} R^{*}(u)(t) W(t) R(u)(t)<+\infty\right\}
$$

with the inner product

$$
\langle u, v\rangle_{W}:=\sum_{t \in \mathcal{I}} R^{*}(v)(t) W(t) R(u)(t),
$$

where the weight function $W(t)$ is a $2 n \times 2 n$ non-negative Hermitian matrix, $R(\cdot)$ is defined by (1.5). Then $L_{W}^{2}(\mathcal{I})$ is a Hilbert space with the inner product $\langle\cdot, \cdot\rangle_{w}$ in the sense that $u=v$ if $\|u-v\|_{W}=0$, where $\|\cdot\|_{W}:=\left(\langle\cdot, \cdot\rangle_{W}\right)^{1 / 2}$.

As stated in the previous section, (1.1) can be converted into (1.3) by (1.2). It is evident that for any $\lambda \in \mathbb{C}$, if $y(t)$ is a solution of (1.1), then $u(t)$ defined by (1.2) is a solution of (1.3) and conversely, if $u(t)$ is a solution of (1.3), then $y(t)=u_{1}(t+1)$ is a solution of (1.1). Moreover, it follows from (1.2) and (1.4) that

$$
\|u\|_{W}^{2}=\sum_{t \in \mathcal{I}} R^{*}(u)(t) W(t) R(u)(t)=\sum_{t \in \mathcal{I}} w(t)|\gamma(t)|^{2}=\|y\|_{w}^{2} .
$$

By $d_{w}(\lambda)$ and $D_{W}(\lambda)$ denote the number of the linearly independent solutions of Equation $(1.1)$ in $l_{w}^{2}(\mathcal{I})$ and system $(1.3)$ in $L_{W}^{2}(\mathcal{I})$, respectively. Then the following result is obtained:

Lemma 2.1. $D_{W}(\lambda)=d_{w}(\lambda)$ for any $\lambda \in \mathbb{C}$.

Denote $d_{+}=d_{w}(i)$ and $d_{-}=d_{w}(-i)$. By Theorem 5.1 of [16], $d_{+}$and $d_{-}$are equal to the positive and negative defect indices of the minimal operator generated by (1.1), respectively. The following result is directly derived from Corollary 4.1 and Theorem 5.4 in [16] and Lemma 2.1:

Lemma 2.2. $n \leq d_{+}, d_{-} \leq 2 n$.

Definition 2.1. Equation (1.1) is called in the $\left(d_{+}, d_{-}\right)$case at $t=+\infty$. In the case that $d_{ \pm}=n$, Equation (1.1) is called in the limit point case at $t=+\infty$; in the case that $d_{ \pm}=$ $2 n$, Equation (1.1) is called in the limit circle case at $t=+\infty$.

Lemma 2.3 [14]. For second-order symmetric difference equation:

$$
c(t+1) y(t+1)+b(t) y(t)+\bar{c}(t) y(t-1)=\lambda w(t) y(t), \quad t \in \mathcal{I}
$$

where $c(t)$ is a complex-valued function and $b(t)$ is a real-valued function, and $w(t)>0$ on $\mathcal{I}$, the positive and negative defect indices $\left(d_{+}, d_{-}\right)$are equal to either $(1,1)$ or $(2,2)$.

2.2 Two equivalent forms of Equation (1.1)

In this section, we formulate two equivalent forms of (1.1).

For convenience, we first introduce the following useful formulae:

$$
\begin{aligned}
& \Delta^{k} \gamma(t)=\nabla^{k} \gamma(t+k)=\sum_{j=0}^{k}(-1)^{k-j} C_{k}^{j} \gamma(t+j), \\
& \Delta^{k}(\gamma(t) z(t))=\sum_{j=0}^{k} C_{k}^{j} \Delta^{j} \gamma(t) \Delta^{k-j} z(t+j),
\end{aligned}
$$

where $C_{k}^{j}=k ! /(j !(k-j) !)$ is the binomial coefficient. 
First, by using (2.2) and (2.3), Equation (1.1) can be rewritten as

$$
\begin{aligned}
& \sum_{j=1}^{n}\left(P_{j}(t+j)+i Q_{j}(t+j)\right) y(t+j)+P_{0}(t) y(t)+\sum_{j=1}^{n}\left(P_{j}(t)-i Q_{j}(t)\right) y(t-j) \\
& \quad=\lambda w(t) y(t), \quad t \in \mathcal{I}
\end{aligned}
$$

where

$$
\begin{aligned}
P_{j}(t) & =(-1)^{j} \sum_{s=j}^{n} \sum_{k=0}^{s-j} C_{s}^{k} C_{s}^{s-j-k} p_{s}(t+k), \quad 1 \leq j \leq n, \\
P_{0}(t) & =\sum_{s=0}^{n} \sum_{k=0}^{s} C_{s}^{k} C_{s}^{s-k} p_{s}(t+k), \\
Q_{j}(t) & =(-1)^{j+1} \sum_{k=j}^{n} C_{k}^{j} q_{k}(t), \quad 1 \leq j \leq n,
\end{aligned}
$$

or

$$
\begin{aligned}
& p_{n}(t)=(-1)^{n} P_{n}(t), \\
& p_{j}(t)=(-1)^{j} P_{j}(t)-\sum_{s=j+1}^{n} \sum_{k=0}^{s-j} C_{s}^{k} C_{s}^{s-j-k} p_{s}(t+k), \quad 0 \leq j \leq n-1, \\
& q_{n}(t)=(-1)^{n+1} Q_{n}(t), \\
& q_{j}(t)=(-1)^{j+1} Q_{j}(t)-\sum_{k=j+1}^{n} C_{k}^{j} q_{k}(t), \quad 1 \leq j \leq n-1 .
\end{aligned}
$$

Equation (2.4) is a equivalent form of Equation (1.1). In addition, it is evident that Equation (2.1) is a special case of Equation (2.4) with $n=1$.

Second, setting

$$
y(t)=w^{-1 / 2}(t) x(t), \quad t \geq 0,
$$

and multiplying $w^{-1 / 2}(t)$ on the both side of (2.4) we get

$$
\begin{aligned}
& \sum_{j=1}^{n}\left(\hat{P}_{j}(t+j)+i \hat{Q}_{j}(t+j)\right) x(t+j)+\hat{P}_{0}(t) x(t)+\sum_{j=1}^{n}\left(\hat{P}_{j}(t)-i \hat{Q}_{j}(t)\right) x(t-j) \\
& \quad=\lambda x(t), \quad t \geq n,
\end{aligned}
$$

where

$$
\begin{aligned}
& \hat{P}_{j}(t)=P_{j}(t) w^{-1 / 2}(t) w^{-1 / 2}(t-j), \quad 0 \leq j \leq n, \\
& \hat{Q}_{j}(t)=Q_{j}(t) w^{-1 / 2}(t) w^{-1 / 2}(t-j), \quad 1 \leq j \leq n .
\end{aligned}
$$

Further, (2.8) can be rewritten as

$$
\begin{aligned}
& \sum_{j=0}^{n}(-1)^{j} \Delta^{j}\left(\hat{p}_{j}(t) \nabla^{j} x(t)\right)+i \sum_{k=1}^{n}\left[(-1)^{k+1} \Delta^{k}\left(\hat{q}_{k}(t) x(t)\right)+\hat{q}_{k}(t) \nabla^{k} x(t)\right] \\
& \quad=\lambda x(t), \quad t \geq n,
\end{aligned}
$$


where $\hat{p}_{j}(t)$ and $\hat{q}_{j}(t)$ are determined by (2.6) with $P_{j}(t), p_{s}(t), Q_{j}(t)$, and $q_{s}(t)$ replaced by $\hat{P}_{j}(t), \hat{p}_{s}(t), \hat{Q}_{j}(t)$, and $\hat{q}_{s}(t)$, respectively. It is evident that $\hat{p}_{j}(t), 0 \leq j \leq n$, and $\hat{q}_{k}(t), 1$ $\leq k \leq n$, are all real-valued, and $\hat{p}_{n}^{2}(t)+\hat{q}_{n}^{2}(t) \neq 0$ for all $t \geq n$.

Equation (2.10) is an another equivalent form of Equation (1.1). It is evident that for any $\lambda \in \mathbb{C}, y(t)$ is a solution of Equation (1.1) if and only if $x(t)=w^{1 / 2}(t) y(t)$ is a solution of Equation (2.10). Moreover, it follows that

$$
\sum_{t=0}^{+\infty} w(t)|y(t)|^{2}<+\infty
$$

if and only if

$$
\sum_{t=n}^{+\infty}|x(t)|^{2}<+\infty
$$

By $d(\lambda)$ denotes the numbers of the linearly independent solutions of Equation (2.10) in $l^{2}(\mathcal{I})$. Then we have that

$$
d(\lambda)=d_{w}(\lambda), \quad \forall \lambda \in \mathbb{C} .
$$

This means that Equation (1.1) has the same positive and negative defect indices as those of Equation (2.10).

\subsection{Sufficient and necessary conditions of limit point case and strong limit point case}

The natural difference operator corresponding to Equation (1.1) is defined by

$$
\mathcal{L}(y)(t):=\sum_{j=0}^{n}(-1)^{j} \Delta^{j}\left(p_{j}(t) \nabla^{j} \gamma(t)\right)+i \sum_{k=1}^{n}\left[(-1)^{k+1} \Delta^{k}\left(q_{k}(t) y(t)\right)+q_{k}(t) \nabla^{k} \gamma(t)\right],
$$

and the bilinear form $[\cdot, \cdot]$ associated with (1.1) is defined by

$$
\begin{aligned}
{[x, y](t) } & :=\sum_{j=1}^{n}\left(\sum_{k=j}^{n}(-1)^{k-j}\left[\Delta^{k-j}\left(p_{k}(t) \nabla^{k} \bar{y}(t)+i q_{k}(t) \bar{y}(t)\right)\right]\right) \Delta^{j-1} x(t-j) \\
& -\sum_{j=1}^{n} \Delta^{j-1} \bar{y}(t-j)\left(\sum_{k=j}^{n}(-1)^{k-j}\left[\Delta^{k-j}\left(p_{k}(t) \nabla^{k} x(t)-i q_{k}(t) x(t)\right)\right]\right) .
\end{aligned}
$$

Then, it follows from [16, Lemma 2.2] that for any $x(t)$ and $y(t)$ defined in $\{t\}_{t=-n}^{+\infty}$ we have

$$
\sum_{t=0}^{m}[\bar{y}(t)(\mathcal{L} x)(t)-(\overline{\mathcal{L} y})(t) x(t)]=\left.[x, y](t)\right|_{t=0} ^{m+1} .
$$

Denote

$$
\mathcal{D}:=\left\{y \in l_{w}^{2}(\mathcal{I}): w^{-} \mathcal{L} y \in l_{w}^{2}(\mathcal{I})\right\},
$$

which is the domain of the maximal operator corresponding to operator $\mathcal{L}$. It yields from (2.11) that $\lim _{t \rightarrow+\infty}[x, y](t)=c$ for any $x, y \in \mathcal{D}$, where $c$ is a finite constant. The following lemma is a direct consequence of [16, Theorem 6.15]. 
Lemma 2.4. Equation (1.1) is in l.p.c. at $t=+\infty$ if and only if for all $x, y \in \mathcal{D}$,

$$
\lim _{t \rightarrow+\infty}[x, y](t)=0
$$

Following the concept of the strong limit point case for singular discrete Hamiltonian system given in [17], we give the following concept:

Definition 2.2. Equation (1.1) is said to be in the strong limit point case (s.l.p.c.) at $t$ $=+\infty$ if for all $x, y \in \mathcal{D}$,

$$
\lim _{t \rightarrow+\infty} \sum_{j=1}^{n} \Delta^{j-1} \bar{\gamma}(t-j)\left(\sum_{k=j}^{n}(-1)^{k-j} \Delta^{k-j}\left[p_{k}(t) \nabla^{k} x(t)-i q_{k}(t) x(t)\right]\right)=0 .
$$

For convenience, denote

$$
S(\gamma)(t):=\sum_{j=1}^{n} \Delta^{j-1} \bar{\gamma}(t-j)\left(\sum_{k=j}^{n}(-1)^{k-j}\left[\Delta^{k-j}\left(p_{k}(t) \nabla^{k} \gamma(t)-i q_{k}(t) y(t)\right)\right]\right) .
$$

The following result is a direct consequence of Theorem 2.1 in [17].

Lemma 2.5. Equation (1.1) is in s.l.p.c. at $t=+\infty$ if and only if $\lim _{t \rightarrow+\infty} S(y)(t)=0$ for all $y \in \mathcal{D}$.

Lemma 2.6. Assume that there exist a constant $c$ and an integer $t_{0} \geq 0$ such that $p_{j}(t)$ $(1 \leq j \leq n)$ and $p_{0}(t)-c w(t)$ are either non-negative for all $t \geq t_{0}$ or non-positive for all $t$ $\geq t_{0}$. Then $\lim _{t \rightarrow+\infty} S(y)(t)$ exists, finite or infinite, for all $y \in \mathcal{D}$.

Proof. Using (2.2) and (2.3), we have that

$$
\begin{aligned}
\Delta S(\gamma)(t)= & \sum_{j=1}^{n} \Delta^{j} \bar{\gamma}(t-j)\left(\sum_{k=j}^{n}(-1)^{k-j} \Delta^{k-j}\left[p_{k}(t) \nabla^{k} \gamma(t)-i q_{k}(t) y(t)\right]\right) \\
& +\sum_{j=1}^{n} \Delta^{j-1} \bar{\gamma}(t-j+1)\left(\sum_{k=j}^{n}(-1)^{k-j} \Delta^{k-j+1}\left[p_{k}(t) \nabla^{k} \gamma(t)-i q_{k}(t) y(t)\right]\right) \\
= & \sum_{j=1}^{n} \nabla^{j} \bar{\gamma}(t)\left(\sum_{k=j}^{n}(-1)^{k-j} \Delta^{k-j}\left[p_{k}(t) \nabla^{k} \gamma(t)-i q_{k}(t) y(t)\right]\right) \\
& -\sum_{j=0}^{n-1} \nabla^{j} \bar{\gamma}(t)\left(\sum_{k=j+1}^{n}(-1)^{k-j} \Delta^{k-j}\left[p_{k}(t) \nabla^{k} \gamma(t)-i q_{k}(t) y(t)\right]\right) \\
= & \sum_{j=1}^{n} p_{j}(t)\left|\nabla^{j} \gamma(t)\right|^{2}+\bar{\gamma}(t)\left(p_{0}(t) y(t)-(\mathcal{L} \gamma)(t)\right) \\
= & \sum_{j=0}^{n} p_{j}(t)\left|\nabla^{j} \gamma(t)\right|^{2}-\bar{\gamma}(t)(\mathcal{L} \gamma)(t) .
\end{aligned}
$$

Summing up above relation from $t_{0}$ to $m$, we get

$$
\begin{aligned}
S(\gamma)(m+1)= & S(\gamma)\left(t_{0}\right)+c \sum_{t=t_{0}}^{m} w(t)|\gamma(t)|^{2}-\sum_{t=t_{0}}^{m} \bar{\gamma}(t)(\mathcal{L} \gamma)(t) \\
& +\sum_{t=t_{0}}^{m}\left(p_{0}(t)-c w(t)\right)|\gamma(t)|^{2}+\sum_{j=1}^{n} \sum_{t=t_{0}}^{m} p_{j}(t)\left|\nabla^{j} \gamma(t)\right|^{2}
\end{aligned}
$$


For any $y \in \mathcal{D}$, it follows from Cauchy's inequality that the second and third items on the right side of (2.13) converge. Since $p_{j}(t)(1 \leq j \leq n)$ and $p_{0}(t)-c w(t)$ are either nonnegative for all $t \geq t_{0}$ or non-positive for all $t \geq t_{0}$, the last two items on the right side of (2.13) are monotonic. Therefore, $\lim _{t \rightarrow+\infty} S(y)(t)$ exists, finite or infinite. The proof is complete.

Remark 2.1. Note that the assertion of Lemma 2.6 in the present article are the same that of [15, Lemma 2.7]. Moreover, we point out that none conditions of Lemma 2.6 impose a restriction on $q_{j}(t), 1 \leq j \leq n$.

\section{Main results}

In this section, several results on the positive and negative defect indices of Equation (1.1) and its equivalent forms; that is, (2.4) and (2.10), are given. It is first shown that for a class of symmetric difference equations in form of (2.4), the positive and negative defect indices are equal; that is $n \leq d_{+}=d_{-} \leq 2 n$, and all values in this range are realized. Next, several sufficient conditions of the limit point case for Equations (2.10) and (1.1) are given. Finally, two criteria of the strong limit point case for Equations (2.10) and (1.1) are established.

\subsection{The range of the positive and negative defect indices}

In this section, we consider a special case of Equation (2.4):

$$
P(t+n) y(t+n)+\bar{P}(t) y(t-n)=\lambda w(t) y(t), \quad t \in \mathcal{I}
$$

where $P(t)$ is a complex-valued function, and $w(t)>0$ in $\mathcal{I}$.

Theorem 3.1. The positive and negative defect indices $d_{ \pm}$of Equation (3.1) satisfy that $n \leq d_{+}=d_{-} \leq 2 n$, and all the values in this range are realized.

Proof. We only prove the assertion holds for $n=2$, and the other cases can be shown similarly.

In the case that $n=2$, Equation (3.1) can be written as

$$
P(t+2) y(t+2)+\bar{P}(t) y(t-2)=\lambda w(t) y(t), \quad t \in \mathcal{I}
$$

It can be divided into the following two second order symmetric difference equations:

$$
\begin{array}{ll}
Q_{1}(t+1) x(t+1)+\bar{Q}_{1}(t) x(t-1)=\lambda w_{1}(t) x(t), & t \in \mathcal{I} \\
Q_{2}(t+1) z(t+1)+\bar{Q}_{2}(t) z(t-1)=\lambda w_{2}(t) z(t), & t \in \mathcal{I}
\end{array}
$$

where

$$
\begin{aligned}
& Q_{1}(t)=P(2 t), \quad w_{1}(t)=w(2 t), \quad x(t)=y(2 t), \\
& Q_{2}(t)=P(2 t+1), \quad w_{2}(t)=w(2 t+1), \quad z(t)=y(2 t+1) .
\end{aligned}
$$

For any given $\lambda \in \mathbb{C}$, let $x(t)$ be a solution of Equation (3.3) and $z(t)$ be a solution of Equation (3.4). Denote

$$
\begin{aligned}
& y^{\prime}(t)= \begin{cases}x(k), & t=2 k, \\
0, & t=2 k+1,\end{cases} \\
& y^{\prime \prime}(t)=\left\{\begin{array}{ll}
0, & t=2 k, \\
z(k), & t=2 k+1,
\end{array} \quad k \in \mathcal{I} .\right.
\end{aligned}
$$


It can be easily verified that $y^{\prime}$ and $y^{\prime \prime}$ are two linearly independent solutions of Equation (3.2). Further, since

$$
\begin{aligned}
\sum_{t \in \mathcal{I}} w(t)\left|y^{\prime}(t)\right|^{2} & =\sum_{t \in \mathcal{I}} w_{1}(t)|x(t)|^{2}, \\
\sum_{t \in \mathcal{I}} w(t)\left|y^{\prime \prime}(t)\right|^{2} & =\sum_{t \in \mathcal{I}} w_{2}(t)|z(t)|^{2},
\end{aligned}
$$

It follows that $y^{\prime} \in l_{w}^{2}(\mathcal{I})$ if and only if $x \in l_{w_{1}}^{2}(\mathcal{I})$, and $y^{\prime \prime} \in l_{w}^{2}(\mathcal{I})$ if and only if $z \in l_{w_{2}}^{2}(\mathcal{I})$. By $d_{ \pm}, d_{ \pm}^{\prime}$ and $d_{ \pm}^{\prime \prime}$ denote the positive and negative defect indices of Equations (3.2), (3.3), and (3.4), respectively. Then it follows that

$$
d_{ \pm}=d_{ \pm}^{\prime}+d_{ \pm}^{\prime \prime} .
$$

On the other hand, one has by Lemma 2.3 that

$$
1 \leq d_{+}^{\prime}=d_{-}^{\prime} \leq 2, \quad 1 \leq d_{+}^{\prime \prime}=d_{-}^{\prime \prime} \leq 2,
$$

and all the values in this range can be realized. Inserting (3.6) into (3.5), one has that

$$
2 \leq d_{+}=d_{-} \leq 4 .
$$

and all the values in this range can be realized.

For the general Equation (3.1), we can write it into $n$ second-order symmetric difference equations with complex-valued coefficients. The positive and negative defect indices are equal to the sum of those of the $n$ second-order symmetric difference equations, respectively. Then by Lemma 2.3 one can prove the assertion in Theorem 3.1. The proof is complete.

Example 3.1. Let $w_{1}(t) \equiv 1$ for $t \in \mathcal{I}$. It has been shown in [14] that for Equation (3.3), if $Q_{1}(t) \equiv 1$ for $t \in \mathcal{I}$, then Equation (3.3) is in the limit point case at $t=+\infty$; if $Q_{1}(t)=-4 t+4^{t} i$ for $t \in \mathcal{I}$, then Equation (3.3) is in the limit circle case at $t=+\infty$. Thus, if

$$
P(t) \equiv 1, \quad t \in \mathcal{I},
$$

then the positive and negative defect indices of Equation (3.2) are (2, 2); if

$$
P(t)= \begin{cases}1, \quad t=2 k \\ -4^{k}+4^{k} i, & t=2 k+1,\end{cases}
$$

then the positive and negative defect indices of Equation (3.2) are (3, 3); if

$$
P(t)=\left\{\begin{array}{l}
-4^{k}+4^{k} i, t=2 k \\
-4^{k}+4^{k} i, t=2 k+1
\end{array}\right.
$$

then the positive and negative defect indices of Equation (3.2) are $(4,4)$. This example shows that the positive and negative defect indices of Equation (3.1) satisfies the inequality $n \leq d_{+}=d_{-} \leq 2 n$ and all values in this range are realized.

\subsection{Criteria of the limit point and the strong limit point cases}

In this section, we give some sufficient conditions for the limit point case and the strong limit point case.

First, we consider the criteria of the limit point case. Similarly to the notations in Section 2, we introduce the following notations for Equation (2.10): 


$$
\begin{aligned}
\mathcal{L}_{1}(\gamma)(t):= & \sum_{j=0}^{n}(-1)^{j} \Delta^{j}\left(\hat{p}_{j}(t) \nabla^{j} y(t)\right) \\
& +i \sum_{k=1}^{n}\left[(-1)^{k+1} \Delta^{k}\left(\hat{q}_{k}(t) y(t)\right)+\hat{q}_{k}(t) \nabla^{k} \gamma(t)\right], \\
{[x, y]_{1}(t):=} & \sum_{j=1}^{n}\left(\sum_{k=j}^{n}(-1)^{k-j}\left[\Delta^{k-j}\left(\hat{p}_{k}(t) \nabla^{k} \bar{\gamma}(t)+i \hat{q}_{k}(t) \bar{\gamma}(t)\right)\right]\right) \Delta^{j-1} x(t-j) \\
& -\sum_{j=1}^{n} \Delta^{j-1} \bar{\gamma}(t-j)\left(\sum_{k=j}^{n}(-1)^{k-j}\left[\Delta^{k-j}\left(\hat{p}_{k}(t) \nabla^{k} x(t)-i \hat{q}_{k}(t) x(t)\right)\right]\right), \\
S_{1}(y)(t):= & \sum_{j=1}^{n} \Delta^{j-1} \bar{\gamma}(t-j)\left(\sum_{k=j}^{n}(-1)^{k-j}\left[\Delta^{k-j}\left(\hat{p}_{k}(t) \nabla^{k} \gamma(t)-i \hat{q}_{k}(t) y(t)\right)\right]\right), \\
\mathcal{D}_{1}:= & \left\{y \in l^{2}(\mathcal{I}): \mathcal{L}_{1} y \in l^{2}(\mathcal{I})\right\} .
\end{aligned}
$$

Theorem 3.2. Assume that there exist a non-negative function $\sigma(t)$ defined on $\mathcal{I}$, a constant $M>0$ and an integer $t_{0} \geq 0$ such that

$$
\begin{aligned}
& \sum_{t \in \mathcal{I}} \sigma(t)=+\infty, \\
& \sigma(t)\left|\Delta^{l} \hat{p}_{j}(t)\right| \leq M, \quad \sigma(t)\left|\Delta^{l} \hat{q}_{j}(t)\right| \leq M, \quad t \geq t_{0},
\end{aligned}
$$

for all $1 \leq j \leq n$ and $0 \leq l \leq j-1$. Then Equation (2.10) is in l.p.c. at $t=+\infty$.

Proof. First, we mention that the main idea of the proof is the same as that of [15, Theorem 3.3]. By Lemma 2.4, it suffices to show $\lim _{t \rightarrow+\infty}[x, y]_{1}(t)=0$ for all $x, y \in \mathcal{D}_{1}$. By the discussion before Lemma 2.4, $\lim _{t \rightarrow+\infty}[x, y]_{1}(t)$ exists, finite, for all $x, y \in \mathcal{D}_{1}$. Suppose that there exist $x, y \in \mathcal{D}_{1}$ such that $\lim _{t \rightarrow+\infty}[x, y]_{1}(t)=c \neq 0$. Then there exists $T_{0} \geq t_{0}$ such that $\left|[x, y]_{1}(t)\right| \geq|c| / 2>0$ for all $t \geq T_{0}$. It follows from condition (3.7) that

$$
\sum_{t=T_{0}}^{+\infty} \sigma(t)\left|[x, y]_{1}(t)\right| \geq \frac{|c|}{2} \sum_{t=T_{0}}^{+\infty} \sigma(t)=+\infty .
$$

On the other hand, we have from (2.3) that

$$
\begin{aligned}
\left|[x, y]_{1}(t)\right| \leq & \sum_{j=1}^{n}\left|\Delta^{j-1} x(t-j)\right| \sum_{k=j}^{n} \sum_{s=0}^{k-j} C_{k-j}^{s}\left|\Delta^{s} \hat{p}_{k}(t)\right|\left|\Delta^{2 k-j-s} y(t+s-k)\right| \\
& +\sum_{j=1}^{n}\left|\Delta^{j-1} x(t-j)\right| \sum_{k=j}^{n} \sum_{s=0}^{k-j} C_{k-j}^{s}\left|\Delta^{s} \hat{q}_{k}(t)\right|\left|\Delta^{k-j-s} \gamma(t+s)\right| \\
& +\sum_{j=1}^{n}\left|\Delta^{j-1} \gamma(t-j)\right| \sum_{k=j}^{n} \sum_{s=0}^{k-j} C_{k-j}^{s}\left|\Delta^{s} \hat{p}_{k}(t)\right|\left|\Delta^{2 k-j-s} x(t+s-k)\right| \\
& +\sum_{j=1}^{n}\left|\Delta^{j-1} \gamma(t-j)\right| \sum_{k=j}^{n} \sum_{s=0}^{k-j} C_{k-j}^{s}\left|\Delta^{s} \hat{q}_{k}(t)\right|\left|\Delta^{k-j-s} x(t+s)\right|,
\end{aligned}
$$


which, together with condition (3.8) and Cauchy's inequality, implies that

$$
\begin{aligned}
\sigma(t)\left|[x, y]_{1}(t)\right| & \leq M \sum_{j=1}^{n} \sum_{k=j}^{n} \sum_{s=0}^{k-j} C_{k-j}^{s}\left(2\left|\Delta^{j-1} x(t-j)\right|^{2}+2\left|\Delta^{j-1} y(t-j)\right|^{2}\right. \\
& +\left|\Delta^{k-j-s} \gamma(t+s)\right|^{2}+\left|\Delta^{k-j-s} x(t+s)\right|^{2} \\
& \left.+\left|\Delta^{2 k-j-s} \gamma(t+s-k)\right|^{2}+\left|\Delta^{2 k-j-s} x(t+s-k)\right|^{2}\right) .
\end{aligned}
$$

Since $x, y \in \mathcal{D}_{1},\left|\Delta^{k} x\right|$ and $\left|\Delta^{k} \bar{y}\right|$ belong to $l^{2}(\mathcal{I})$ for any $k \geq 0$. Hence,

$$
\sum_{t=T_{0}}^{+\infty} \sigma(t)\left|[x, y]_{1}(t)\right|<+\infty
$$

This is a contradiction with (3.9). Therefore, $\lim _{t \rightarrow+\infty}[x, y]_{1}(t)=0$ holds for all $x, y \in \mathcal{D}_{1}$. By Lemma 2.4, Equation (2.10) is in l.p.c. at $t=+\infty$.

Reversing the transformation from (1.1) to (2.10), we get the following result:

Theorem 3.3. Assume that there exist a non-negative function $\sigma(t)$ defined on $\mathcal{I}$, a constant $M>0$ and an integer $t_{0} \geq n$ such that $\sigma(t)$ satisfies condition (3.7), and

$$
\begin{aligned}
& \sigma(t)\left|\Delta^{m}\left[p_{s}(t+k+v) w^{-1 / 2}(t+v) w^{-1 / 2}(t-j+v)\right]\right| \leq M \\
& \sigma(t)\left|\Delta^{b}\left[q_{s}(t) w^{-1 / 2}(t) w^{-1 / 2}(t-j)\right]\right| \leq M, \quad t \geq t_{0}
\end{aligned}
$$

for all $1 \leq s \leq n, 1 \leq j \leq s, 0 \leq k \leq s-j, 0 \leq v \leq j-1,0 \leq m \leq j-1-v$, and $0 \leq b \leq j$ - 1. Then Equation (1.1) is in l.p.c. at $t=+\infty$.

Proof. Since Equations (1.1) and (2.10) have the same limit case at $t=+\infty$ under the transformation (2.7), it suffices to show that conditions (3.10) and (3.11) can imply that (3.8) holds. By the proof of Theorem 3.4 in [15], condition (3.10) implies that the first condition in (3.8) holds. So, it remains to show that condition (3.11) can imply that the second condition in (3.8) holds.

From (2.5), (2.6), and (2.9) we have

$$
\begin{aligned}
& \hat{q}_{n}(t)=(-1)^{n-1} q_{n}(t) w^{-1 / 2}(t) w^{-1 / 2}(t-n), \\
& \hat{q}_{j}(t)=\sum_{k=j}^{n} C_{k}^{j} q_{k}(t) w^{-1 / 2}(t) w^{-1 / 2}(t-j)-\sum_{k=j+1}^{n} C_{k}^{j} \hat{q}_{k}(t), \quad 1 \leq j \leq n-1 .
\end{aligned}
$$

When $s=j=n$, it follows from (3.11) that

$$
\sigma(t)\left|\Delta^{b}\left[q_{n}(t) w^{-1 / 2}(t) w^{-1 / 2}(t-n)\right]\right| \leq M, \quad 0 \leq b \leq n-1,
$$

which together with (3.12) yields that

$$
\sigma(t)\left|\Delta^{b} \hat{q}_{n}(t)\right| \leq M, \quad 0 \leq b \leq n-1 .
$$

When $s=j=n-1$, it follows from (3.11) that

$$
\sigma(t)\left|\Delta^{b}\left[q_{n-1}(t) w^{-1 / 2}(t) w^{-1 / 2}(t-n+1)\right]\right| \leq M, \quad 0 \leq b \leq n-2 .
$$


When $s=n$ and $j=n-1$, (3.11) implies that

$$
\sigma(t)\left|\Delta^{b}\left[q_{n}(t) w^{-1 / 2}(t) w^{-1 / 2}(t-n+1)\right]\right| \leq M, \quad 0 \leq b \leq n-2 .
$$

Inserting (3.14)-(3.16) into (3.13) with $j=n-1$, we have

$$
\sigma(t)\left|\Delta^{b} \hat{q}_{n-1}(t)\right| \leq M\left(\sum_{k=n-1}^{n} C_{k}^{n-1}+1\right), \quad 0 \leq b \leq n-2 .
$$

With a similar argument one can conclude that

$$
\sigma(t)\left|\Delta^{b} \hat{q}_{s}(t)\right| \leq M\left(\sum_{k=s}^{n} C_{k}^{s}+\sum_{k=s+1}^{n} C_{k}^{s}\right)
$$

for all $1 \leq s \leq n, 0 \leq b \leq s-1$ by using (3.13). Therefore, the conditions for $q_{k}$ in (3.8) hold. By Theorem 3.3, Equation (2.10) is in l.p.c. at $t=+\infty$, which is equivalent to that Equation (1.1) is in l.p.c. at $t=+\infty$. The proof is complete.

Note that if there exist a constant $K>0$ and an integer $t_{0} \geq 0$ such that $|f(t)| \leq K t$ for $t \geq t_{0}$, then there exists a constant $N>0$ such that $\left|\Delta^{m} f(t+v)\right| \leq N t$ for $t \geq t_{0}+1$ and $0 \leq m, v \leq n$. Thus the following result is a direct consequence of Theorem 3.4 by taking $\sigma(t)=t^{-1}$ :

Corollary 3.1. If there exist a constant $M>0$ and an integer $t_{0} \geq n$ such that

$$
\begin{aligned}
& \left|p_{s}(t+k)\right| \leq M t w^{1 / 2}(t) w^{1 / 2}(t-j), \\
& \left|q_{s}(t)\right| \leq M t w^{1 / 2}(t) w^{1 / 2}(t-j), \quad j \geq t_{0},
\end{aligned}
$$

for all $1 \leq s \leq n, 1 \leq j \leq s$ and $0 \leq k \leq s-j$, then Equation (1.1) is in l.p.c. at $t=+\infty$.

Remark 3.1. In the case that $n=1$, the conditions of Theorem 3.3 are equivalent to that of [14, Theorem 3.1]; that is

$$
\sum_{t \in \mathcal{I}} \frac{(w(t) w(t+1))^{1 / 2}}{\sqrt{p_{1}^{2}(t+1)+q_{1}^{2}(t+1)}}=+\infty
$$

In fact, the conditions of Theorem 3.3 in the case that $n=1$ are

$$
\begin{aligned}
& \sum_{t \in \mathcal{I}} \sigma(t)=+\infty \\
& \sigma(t)\left|p_{1}(t) w^{-1 / 2}(t) w^{-1 / 2}(t-1)\right| \leq M \\
& \sigma(t)\left|q_{1}(t) w^{-1 / 2}(t) w^{-1 / 2}(t-1)\right| \leq M, \quad t \geq t_{0} .
\end{aligned}
$$

If (3.19) holds, then (3.20) holds with

$$
\sigma(t)=\frac{(w(t) w(t-1))^{1 / 2}}{\sqrt{p_{1}^{2}(t)+q_{1}^{2}(t)}}
$$

and $M=1$. On the other hand, suppose that (3.20) holds. Then it follows from the second and third conditions in (3.20) that 


$$
\frac{(w(t) w(t-1))^{1 / 2}}{\sqrt{p_{1}^{2}(t)+q_{1}^{2}(t)}} \geq \frac{(w(t) w(t-1))^{1 / 2}}{\left|p_{1}(t)\right|+\left|q_{1}(t)\right|} \geq \frac{\sigma(t)}{2 M},
$$

which, together with the first condition in (3.20), implies that (3.19) holds.

At the end of this section, two criteria of the strong limit point case for Equations (2.10) and (1.1) are established, respectively.

Theorem 3.4. If $\hat{p}_{j}, 0 \leq j \leq n$, and $\hat{q}_{k}(t), 1 \leq k \leq n$, satisfy all the conditions in Theorem 3.2 and Lemma 2.6, then Equation (2.10) is in s.l.p.c. at $t=+\infty$.

Proof. Since $q_{j}(t), 0 \leq j \leq n$, satisfy the conditions in Lemma 2.6, $\lim _{t \rightarrow+\infty} S_{1}(y)(t)$ exists, finite or infinite, for all $y \in \mathcal{D}_{1}$. With a similar argument to that used in the proof of Theorem 3.2, it follows that $\lim _{t \rightarrow+\infty} S_{1}(y)(t)=0$ for all $y \in \mathcal{D}_{1}$. By Lemma 2.5, (2.10) is in s.l.p.c. at $t=+\infty$. The proof is complete.

Theorem 3.5. If $p_{j}(t), 0 \leq j \leq n, q_{k}(t), 1 \leq k \leq n$, and $w(t)$ satisfy all the conditions in Theorem 3.3 and Lemma 2.6, then Equation (1.1) is in s.l.p.c. at $t=+\infty$.

\section{Acknowledgements}

This research was supported by the NNSF of China (Grant 11071143, 11101241), and the NNSF of Shandong Province (Grant J10LA62).

\section{Competing interests}

The author declares that she has no competing interests.

Received: 11 November 2011 Accepted: 7 March 2012 Published: 7 March 2012

\section{References}

1. Naimark, MA: Linear Differential Operators, Part II, Linear Differential Operators in Hilbert Space. Ungar Publ. Co., New York (1968)

2. Weidmann, J: Linear Operators in Hilbert Spaces. Springer-Verlag, New York (1980)

3. Coddington, EA: Extension theory of formally normal and symmetric subspaces. Mem Am Math Soc. 134, 1-80 (1973)

4. Glazman, IM: On the theory of singular differential operators. Uspehi Math Nauk 40, 102-135 (1950). English translation in Am. Math. Soc. Transl. Ser. 1 4, 331-372 (1962)

5. Devinatz, A: The deficiency index problem for ordinary self-adjoint differential operators. Bull Am Math Soc. 79 1109-1127 (1973). doi:10.1090/S0002-9904-1973-13348-8

6. Mcleod, JB: The number of integrable-square solutions of ordinary differential equations. Quart J Math Oxford. 17(2):285-290 (1966)

7. Kogan, VI, Rofe-Beketov, FS: On the question of the deficiency indices of differential operators with complex coefficients. Proc Royal Soc Edin. 72, 281-298 (1973)

8. Kogan, VI, Rofe-Beketov, FS: On sqare-integrable solutions of symmetric systems of differential equations of arbitrary order. Proc Royal Soc Edin. 74, 5-40 (1974)

9. Atkinson, FV: Discrete and Continuous Boundary Problems. Academic Press, Inc., New York (1964)

10. Chen, J, Shi, Y: The limit circle and limit point criteria for second-order linear difference equations. Comput Math Appl. 42, 465-476 (2001). doi:10.1016/50898-1221(01)00170-5

11. Clark, SL: A spectral analysis for self-adjoint operators generated by a class of second order difference equations. J Math Anal Appl. 197, 267-285 (1996). doi:10.1006/jmaa.1996.0020

12. Hinton, DB, Lewis, RT: Spectral analysis of second order difference equations. J Math Anal Appl. 63, $421-438$ (1978). doi:10.1016/0022-247X(78)90088-4

13. Jirari, A: Second-order Sturm-Liouville difference equations and orthogonal polynomials. Mem Am Math Soc. 542, 1-136 (1995)

14. Sun, H, Shi, Y: Limit-point and limit-circle criteria for singular second-order linear difference equations with complex coefficients. Comput Math Appl. 52, 539-554 (2006). doi:10.1016/j.camwa.2006.08.024

15. Ren, G, Shi, Y: The defect index of singular symmetric linear difference equations with real coefficients. Proc Am Math Soc. 138, 2463-2475 (2010). doi:10.1090/S0002-9939-10-10253-6

16. Shi, Y: Weyl-Titchmarsh theory for a class of singular discrete linear Hamiltonian systems. Linear Algebra Appl. 416 , 452-519 (2006). doi:10.1016/j.laa.2005.11.025

17. Sun, H, Shi, Y: Strong limit point criteria for a class of singular discrete linear Hamil-tonian systems. J Math Anal Appl. 336, 224-242 (2007). doi:10.1016/j.jmaa.2007.02.058

doi:10.1186/1687-1847-2012-27

Cite this article as: Ren: Defect indices of singular symmetric linear difference equations with complex coefficients. Advances in Difference Equations 2012 2012:27. 